\title{
Combined effects of klotho and soluble CD40 ligand on A549 lung cancer cells
}

\author{
XUELI WANG, BO CHEN, WEI XU, SHIFENG LIU, WEIHONG ZHAO and JIANQING WU \\ Department of Geriatrics, The First Affiliated Hospital of Nanjing Medical University, Nanjing 210029, P.R. China
}

Received October 28, 2010; Accepted January 10, 2011

DOI: 10.3892/or.2011.1178

\begin{abstract}
Although the roles of soluble CD40 ligand (sCD40L) or the klotho gene in lung cancer cells have been studied, little is known about the functions of klotho combined with SCD40L in lung cancer. The present study was designed to investigate the biological effects of klotho combined with sCD40L on the A549 lung cancer cell line (CD40 positive) and their possible mechanisms. Lung cancer A549 cells were chosen as target cells and CD40 signals were stimulated by soluble CD40 ligand (sCD40L). In this study, we found that klotho, soluble CD40 ligand and their combination can increase cell proliferation inhibition and the apoptosis rate in A549 cells by inhibiting the cell cycle, up-regulating Bax gene expression and (or) down-regulating Bcl-2 gene expression; and their combination has a stronger effect on A549 cell apoptosis and proliferation inhibition compared to klotho or sCD40L alone. These data suggest that the combination of klotho and sCD40L may provide an efficient method to treat non-small cell lung cancer.
\end{abstract}

\section{Introduction}

Lung cancer is one of the common malignant tumors. Its mortality is first in all kinds of malignant tumors, with 5-year survival rate approximately $10 \%$ for non-small cell lung cancer (NSCLC). Although the traditional treatment has made great progress, the overall survival rate of patients with lung cancer has not improved (1). It is urgent to find a new treatment. More and more evidence has accumulated on combined gene therapy on tumors $(2,3)$. Klotho gene was identified as an 'anti-aging' gene in mice which accelerates aging when disrupted and extends life span when overexpressed (4). The human klotho gene is located on chromosome 13q12, spanning over $50 \mathrm{~kb}$ in length. A defect in klotho gene expression leads to multiple

Correspondence to: Professor Jianqing Wu, Department of Geriatrics, the First Affiliated Hospital of Nanjing Medical University, 300 Guangzhou Road, Nanjing 210029, P.R. China

E-mail: jwuny@njmu.edu.cn

Key words: klotho, sCD40L, gene therapy, combined therapy, lung neoplasm aging-like phenotypes, including growth retardation (4), rapid thymic involution (5), and osteopenia (6). There are two forms of klotho protein, secreted and membrane protein. The major transcript in human produces the secreted form, and the extrancellular domain of the membrane-bound form is shed and then secreted (7). Several activities of klotho have been described to date, including retention of the calcium channel TRPV5 (8), binding to fibroblast growth factor receptors (FGFR)1-4, acting as a co-receptor for FGF23 (8), inhibiting insulin/insulin-like growth factor-1 receptors (IGF-1) $(9,10)$ and acting as an inhibitor of the Wnt signaling (11). Recently, klotho was identified as a potential tumor suppressor that inhibits the IGF-1 pathway and/or activates the FGF pathway in human breast cancer (9). Besides, research also showed that functional loss of klotho due to epigenetic silencing may contribute to aberrant activation of the canonical Wnt pathway in cervical carcinoma (12). Our previous study found that the expression of klotho in lung cancer cell line A549 is low, and klotho overexpression inhibits, whereas klotho downregulation enhances, lung cancer cell growth (13). All of these studies prove that klotho gene is a potential tumor suppressor. CD40L (also called CD154, TRAP-1, TBA), belonging to TNF superfamily, is a $39-\mathrm{kDa}$ memberane protein that has been first identified on CD4 ${ }^{+} \mathrm{T}$ cells. CD40L has been found on the surface membrane of activated platelets from where a soluble form of CD40L can be released into the circulation (14). CD40L contains a total of 261 amino acids, of which the 46 to 261 amino acids in the extracellular can be cleaved into a form of soluble CD40L, and the 115 to 261 amino acids are CD40 receptor binding domain (RBD) (15). CD40, expressed on different cell types, including mature $\mathrm{T}$ cells, $\mathrm{B}$ cells, monocyte/macrophages, is a member of the TNF receptor superfamily $(15,16)$. Interaction of CD40L-CD40 is crucial for adaptive immune responses. Humans with genetic mutations of CD40L, rendering the protein incapable of binding to CD40, have been found to have reduced circulating levels of immunoglobulins and defects in $\mathrm{T}$ cell function and cell-mediated immunity (16). In addition to its expression in normal lymphoid cells, CD40 is also found in a variety of malignant cells $(17,18)$. However, in marked contrast to the proliferative effect of CD40 ligation on normal B lymphoid cells, researchers still hold different views toward the effect of CD40 ligation on malignant cells. Johnson et al and Clod et al showed that SCD40L-CD40 can promote blood system tumor cells proliferation and tolerance to chemotherapy in 
Hodgkin lymphoma or poorly differentiated B cell malignancies $(19,20)$. At the same time, the growth inhibitory effect has noted in various solid neoplasmas treated with a soluble form or membrane-presented CD40L, including non-small cell lung cancer (21-23), breast cancer (24), epithelial cells (25), ovarian cancer (26), and bladder cancer (27). Since both klotho and sCD40L gene can inhibit the development of lung cancer, and both of them can regulate the cellular immunity and humoral immunity, we hypothesized that the klotho gene combined with SCD40L can suppress tumor growth better than klotho gene or sCD40L alone. In this study, we chose CD40-positive lung cancer cell line (A549), transfected with klotho gene and treated with $\mathrm{sCD} 40 \mathrm{~L}$ at the same time, to investigate the biological effect of klotho combined with soluble CD40 ligand on lung cancer cell line A549 and its mechanism.

\section{Materials and methods}

Group. The cells were assigned to 6 experimental groups: a control group, that received normal treatment, a mock group, that treated with transfection reagent, a pCMV6 group, that transfected with an entry vector (pCMV6), a pCMV6MYC-KL group, that transfected with MYC-tagged klotho expression vector (pCMV6-MYC-KL), a sCD40L group, that received sCD40L (2 $\mu \mathrm{g} / \mathrm{ml})$, a pCMV6-MYC-KL+sCD40L group, that transfected with pCMV6-MYC-KL and treated with $\mathrm{SCD} 40 \mathrm{~L}$ at the same time.

Construct. The MYC-tagged klotho expression vector (pCMV6-MYC-KL) and its entry vector (pCMV6) were designed and purchased from OriGene (Rockville, MD, USA).

Cell culture and treatment. The human CD40-positive lung adenocarcinoma cell line A549 was studied. Our laboratory has detected that the expression rate of CD40 molecules on lung cancer A549 cells surface was $48.1 \pm 6.2 \%$. The cells were maintained in Duibecco's modified Eagle's medium (DMEM) containing $10 \%$ fatal bovine serum (FBS), and cultured in a humidified atmosphere of $95 \%$ air and $5 \% \mathrm{CO}_{2}$ at $37^{\circ} \mathrm{C}$. The cells were observed with a binocular inverted microscope everyday and the medium was changed every other day. The dose of sCD40L administered was $2 \mu \mathrm{g} / \mu \mathrm{l}$ as used by other authors (28).

Transfection of A549 cells. A549 cells were transfected with MegaTran 1.0 transfection reagent (OriGene). The cells transfected with pCMV6 and untreated served as control. All the procedures were performed according to the reagent supplier.

MTT viability assay. Cell proliferation was evaluated by a modified3-(4,5-dimethylthiazol-2-yl)-2,5-diphenyltetrazolium bromide (MTT) assay. The test cells were plated in 96-well culture plates (5000 cells/well) and added DMEM containing $10 \%$ fatal bovine serum (FBS) to adjust the volume to $200 \mu 1$. After different culture time, MTT $(20 \mu 1,10 \mathrm{mg} / \mathrm{ml})$ was added. After an additional $4 \mathrm{~h}$ of incubation, the reaction was terminated by removal of the supernatant and addition of $150 \mu 1 \mathrm{DMSO}$ for $10 \mathrm{~min}$. Optical density (OD) of each well was measured at $570 \mathrm{~nm}$ using ELISA reader (Elx808 Bio-Tek Instruments, USA). Cell growth inhibition rate $(\%)=(\mathrm{OD}$ value of control group - OD value of experimental group)/OD value of control group $\times 100 \%$.

Flow cytometric analysis of apoptosis and cell cycle phase. The tested cells were collected by $0.25 \%$ trypsin digestion, washed twice with ice-cold PBS, fixed in $70 \%$ ethanol at $4^{\circ} \mathrm{C}$ for $30 \mathrm{~min}$, incubated with $500 \mu \mathrm{g} / \mathrm{ml}$ Rnase at $37^{\circ} \mathrm{C}$ for $30 \mathrm{~min}$ and then stained with propidium iodide (PI; $50 \mu \mathrm{g} / \mathrm{ml}$ ). Sample DNA content was analyzed by flow cytometry (FACSCalibur, BD Biosciences). The proportion of cells in different cell cycle phase and undergoing apoptosis was calculated as percentages from statistics generated using Cell Quest software (BD Immunocytometry Systems). The experiments were repeated 3 times.

RNA extraction and quantitative real-time PCR. Total cellular RNA was extracted from the A549 cells with TRIzol reagent (Invitrogen) according to the manufacturer's instructions. Gene expressions were detected by the SYBR-Green Real-time PCR kit (Toyobo) according to the manufacturer's instructions. The primer sequences used for RT-PCR amplification were as follows: klotho (104 bp), sense: 5'-GCT CTC AAA GCC CAC TAC TG-3'; antisense: 5'-GCA GCA TAA CGT AGA GGC C-3'; Bax (91 bp), sense: 5'-ATG CGT CCA CCA AGA AGC-3'; antisense: 5'-ACG GCG GCA ATC ATC CTC; Bcl-2 (402 bp), sense: 5'-ATA GCT GAT TCG ACG TTT TGC C-3'; antisense: 5'-ACA ACA TCG CCC TGT CCC TGT GGA TGA C-3'; $\beta$-actin (205 bp), sense: 5'-TGA CGT GGA CAT CCG CAA AG-3'; antisense: 5'-CTG GAA GGT GGA CAG CGA GG-3' (Invitrogen). The relative levels of gene mRNA transcripts were normalized to the control $\beta$-actin. PCR consisted of initial denaturation at $94^{\circ} \mathrm{C}$ for $5 \mathrm{~min}$, followd by 30 reaction cycles $\left(30 \mathrm{sec}\right.$ at $94^{\circ} \mathrm{C}, 30 \mathrm{sec}$ at $60^{\circ} \mathrm{C}$, and $30 \mathrm{sec}$ at $72^{\circ} \mathrm{C}$ ) and a final cycle at $72^{\circ} \mathrm{C}$ for $10 \mathrm{~min}$.

Statistical analysis. Statistical analysis was performed using SPSS software. Data were expressed as mean \pm SD. Unpaired Student's t-test with two tails was used to determine the significance of results. A value of $\mathrm{P}<0.05$ was considered as statistically significant difference.

\section{Results}

Klotho expression was analyzed, using RT-PCR in A549 cell line. To determine the effect of overexpression of klotho in A549 and 293T cells after transfected with klotho and to detect whether SCD40L can affect the transfection efficiency, the cells were transfected with pCMV6, pCMV6-MYC-KL, respectively treated with $\mathrm{SCD} 40 \mathrm{~L}$ or not at the same time. The results revealed that the levels of klotho mRNA transcripts were highly elevated in pCMV6-MYC-KL-transfected cells when compared with pCMV6, and adding sCD40L at the same time did not affect the transfection efficiency (Fig. 1).

Increased growth inhibition following overexpression of klotho combined with sCD40L in A549. MTT was used to detect the combined effect on A549 cell proliferation. The 

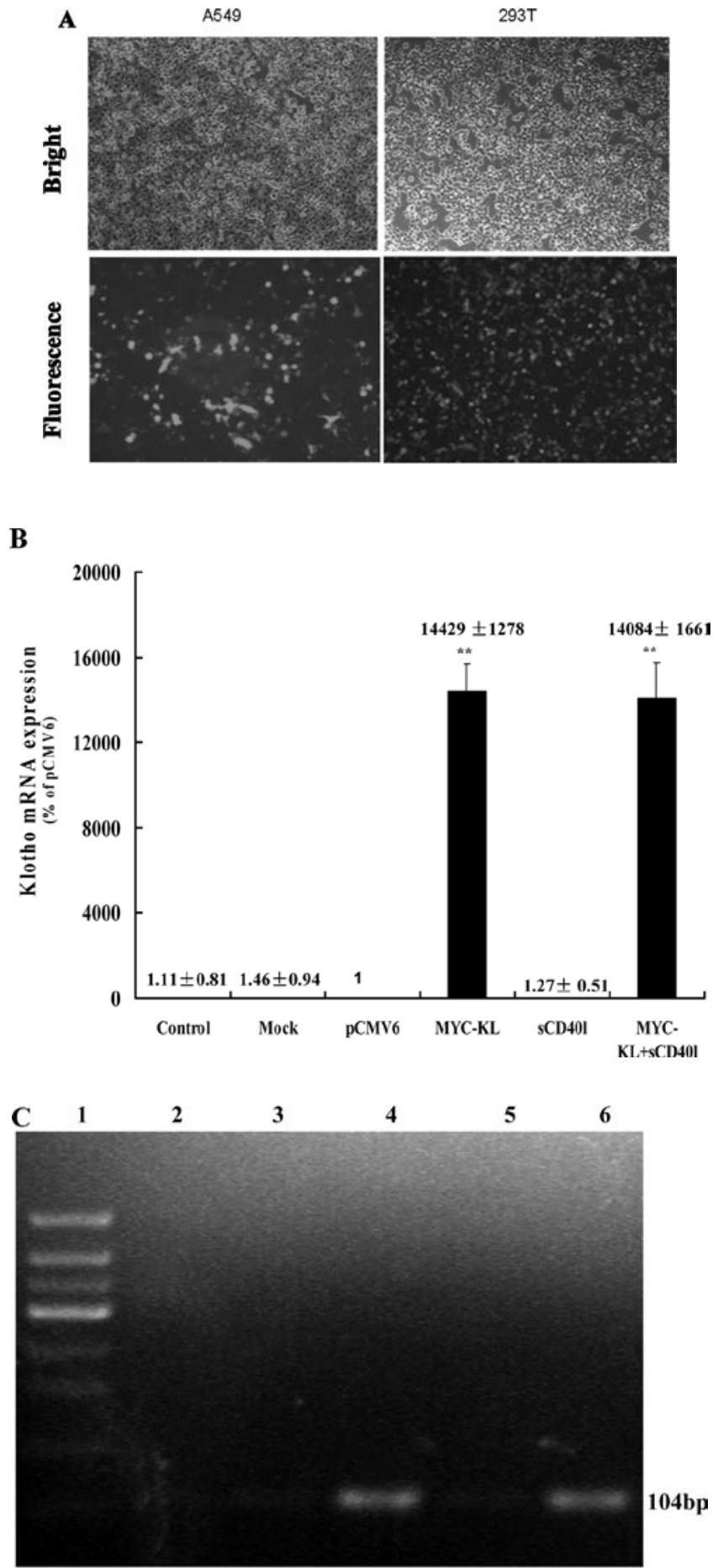

1:Marker 2: Control 3:pCMV6 4:pCMV6-MYC-KL 5:sCD40IL 6:pCMV6-MYC-KL +sCD40L

Figure 1. (A) A549 and $293 \mathrm{~T}$ were transfected with a standard DNA plasmid carrying full-length cDNA encoding for GFP. Images were taken $48 \mathrm{~h}$ post-transfection. (B) Klotho mRNA expression after transfection with pCMV6-MYC-KL by RT-PCR. A549 cells were transfected with an entry vector pCMV6, pCMV6-MYC-KL, pCMV6-MYC-KL combined with sCD40L, and were treated with sCD40L only $\left({ }^{* *} \mathrm{P}<0.01\right.$, vs. pCMV6). (C) Electrophoresis of klotho cDNA outcome from RT-PCR production. Klotho cDNA is $104 \mathrm{bp}$, which is consistent with our expectation.

results showed that: compared with pCMV6 group, klotho gene significantly inhibited the proliferation of lung cancer A549 cells (vs. pCMV6, ${ }^{*} \mathrm{P}<0.05$ ), and the growth inhibition
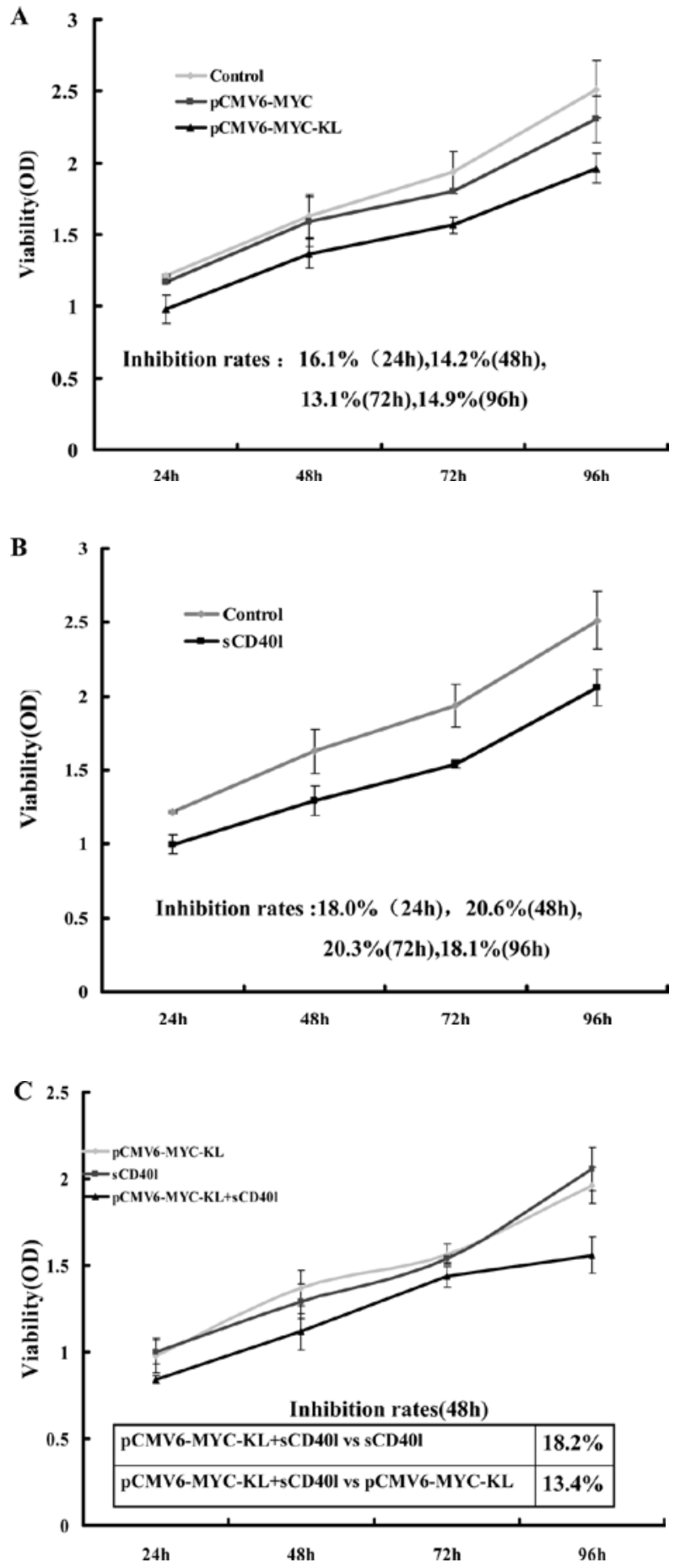

Figure 2. The effect of klotho combined with sCD40L on A549 cell proliferation. A549 cells were measured at $570 \mathrm{~nm}$ using ELISA reader at 24, 48, 72 and $96 \mathrm{~h}$ after treated. (A) The effect of klotho gene on A549 cell proliferation (vs. pCMV6, $\mathrm{P}<0.05$ ); (B) the effect of sCD40L on A549 cells proliferation (vs. control, $\mathrm{P}<0.05$ ); (C) the effect of klotho gene combined with sCD40L on A549 cell proliferation (vs. sCD40L, P<0.05; vs. pCMV6MYC-KL, P<0.05).

rates were: $16.1 \%(24 \mathrm{~h}), 14.2 \%(48 \mathrm{~h}), 13.1 \%(72 \mathrm{~h})$ and $14.9 \%$ (96 h) (Fig. 2A). sCD40L can also inhibit A549 cells proliferation, compared with the control group (vs. control ${ }^{*} \mathrm{P}<0.05$ ), and cell growth inhibition rates were: $18.0 \%$ (24 h), $20.6 \%$ (48 h), 20.3\% (72 h) and 18.1\% (96 h) (Fig. 2B). Besides these, compared with MYC-KL group or sCD40L group alone, the 
A

\begin{tabular}{lccc}
\multicolumn{1}{c}{ Cell cycle distribution } & & & \\
\hline Group & G0/G1 & G2/M & S \\
\hline Control & $58.65 \pm 5.69$ & $13.99 \pm 0.98$ & $27.36 \pm 0.96$ \\
& & & \\
Mock & $59.65 \pm 2.16$ & $14.61 \pm 0.61$ & $25.57 \pm 2.59$ \\
pCMV6 & $59.82 \pm 5.98$ & $13.87 \pm 2.18$ & $27.15 \pm 2.74$ \\
& & & \\
pCMV6-MYC-KL & $63.60 \pm 0.64^{\mathrm{a}}$ & $9.99 \pm 3.98$ & $25.76 \pm 3.33$ \\
sCD40I & & & \\
& & & \\
pCMV6-MYC-KL+sCD40I & $65.00 \pm 4.38^{\mathrm{c}}$ & $7.92 \pm 1.59^{\mathrm{c}}$ & $27.07 \pm 3.90$
\end{tabular}

B
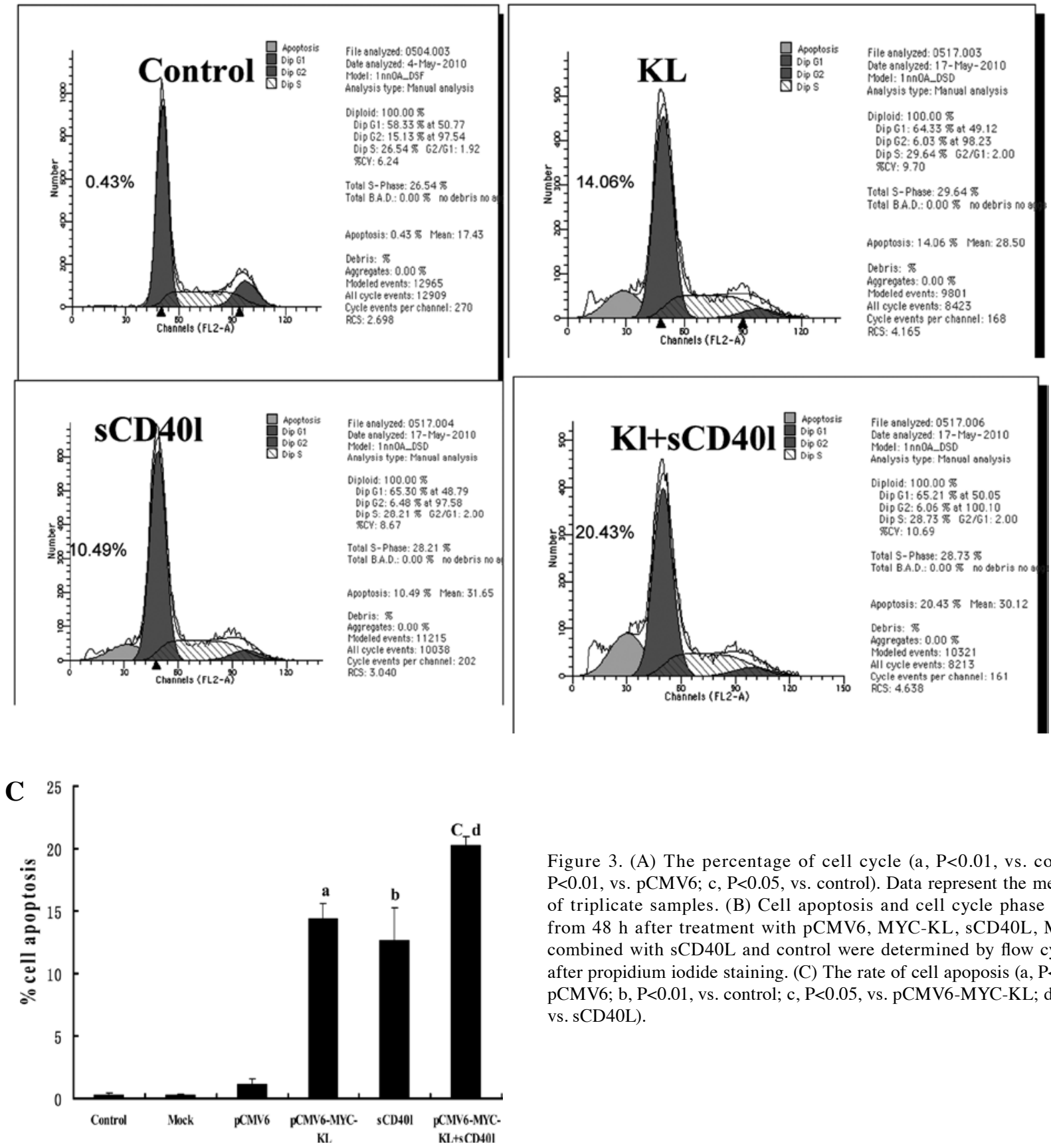

Figure 3. (A) The percentage of cell cycle (a, $\mathrm{P}<0.01$, vs. control; b, $\mathrm{P}<0.01$, vs. pCMV6; c, $\mathrm{P}<0.05$, vs. control). Data represent the mean $\pm \mathrm{SD}$ of triplicate samples. (B) Cell apoptosis and cell cycle phase outcome from $48 \mathrm{~h}$ after treatment with pCMV6, MYC-KL, sCD40L, MYC-KL combined with $\mathrm{SCD} 40 \mathrm{~L}$ and control were determined by flow cytometry after propidium iodide staining. (C) The rate of cell apoposis (a, $\mathrm{P}<0.01$, vs. pCMV6; b, $\mathrm{P}<0.01$, vs. control; c, $\mathrm{P}<0.05$, vs. pCMV6-MYC-KL; d, $\mathrm{P}<0.05$, vs. SCD40L). 

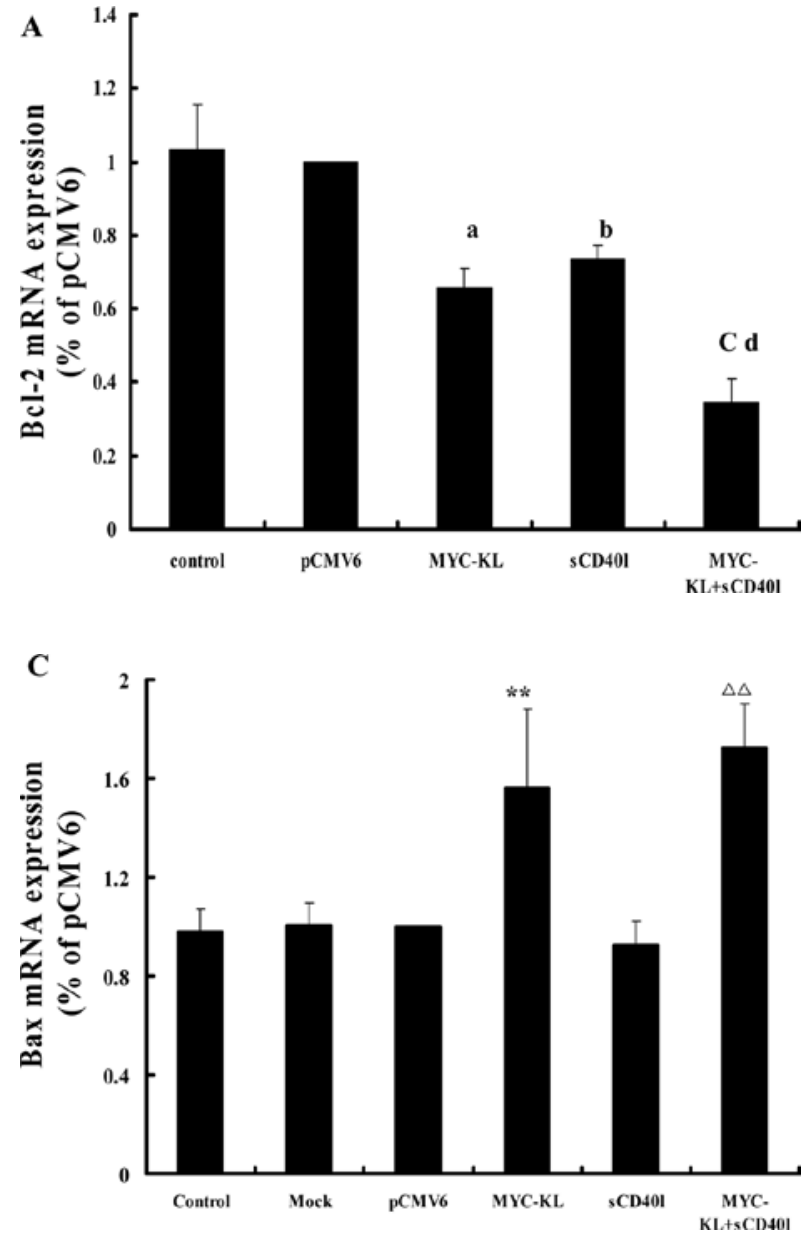
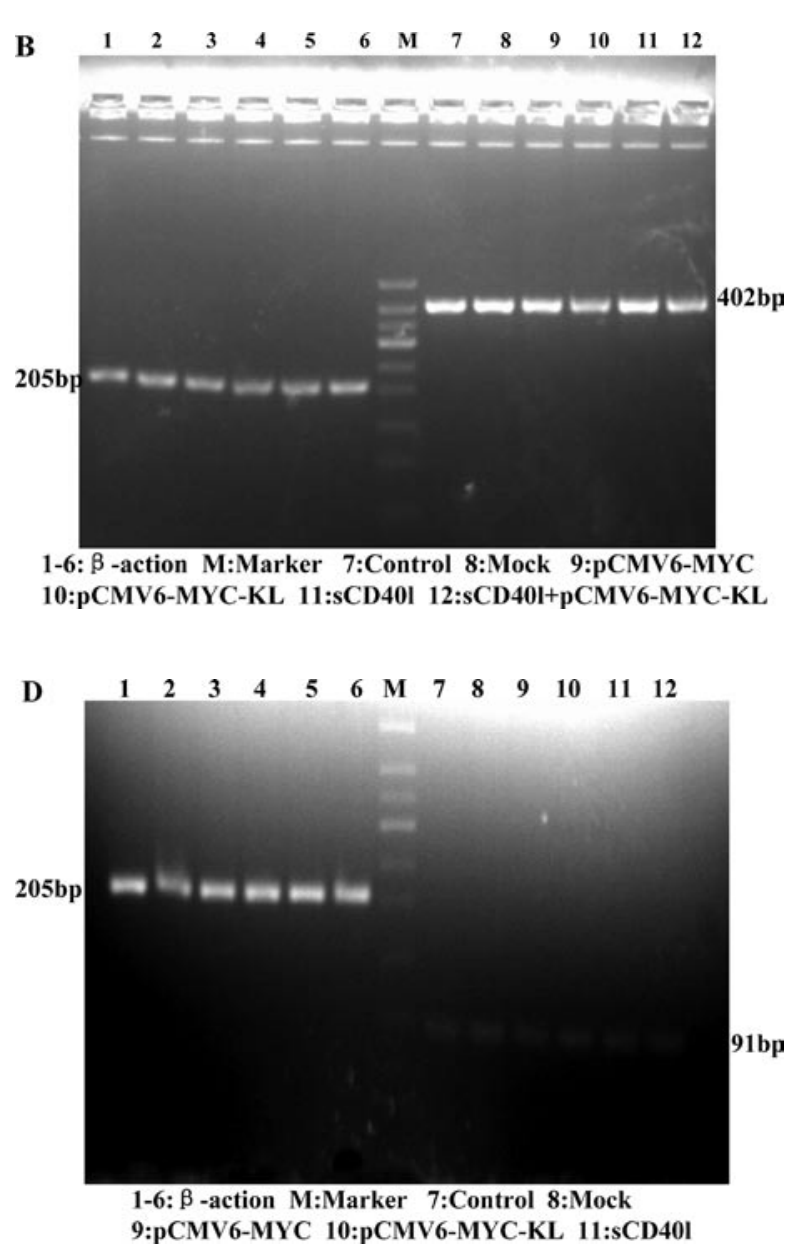

Figure 4. Apoptosis-related genes Bax and Bcl-2 expression. (A) Bcl-2 mRNA expression at 48 h after treatment (a, P<0.05, vs. pCMV6; b, P<0.05, vs. control; c, $\mathrm{P}<0.05$, vs. sCD40L; d, $\mathrm{P}<0.05$, vs. MYC-KL). (B) Electrophoresis of Bcl-2 cDNA outcome from RT-PCR production, and Bcl-2 cDNA was 400 bp. (C) Bax mRNA expression at $48 \mathrm{~h}$ after treatment $\left({ }^{* *} \mathrm{P}<0.01\right.$, vs. pCMV6 or sCD40L; $\triangle \mathrm{P}<0.01$, vs. control or sCD40L or pCMV6). (D) Electrophoresis of Bax cDNA outcome from RT-PCR production, and Bax cDNA was $91 \mathrm{bp}$.

combination group can increase growth inhibition of A549 cells (vs. sCD40L, P<0.05; vs. pCMV6-MYC-KL, P<0.05). Cell growth inhibition rates increased about $18.2 \%$ compared with MYC-KL group and $13.4 \%$ compared with sCD40L group at $48 \mathrm{~h}$ (Fig. 2C). The MYC-KL group and sCD40L group did not have significant differences. These data show that a combination treatment with MYC-KL and sCD40L can enhance the inhibition effect on A549 cell proliferation.

Direct anti-tumor activity of klotho gene combined with ${ }_{s C D} 40 L$. To verify whether klotho gene combined with SCD40L can increase the rate of apoptosis and cell cycle inhibition more effectively than transfection with klotho vector or treatment with SCD40L alone, the cell apoptosis and cell cycle were evaluated by flow cytometry analysis (Fig. 3B). The percentage of G0/G1 phase cells increased in MYC-KL-group and sCD40L-group compared with pCMV-group and controlgroup respectively $(\mathrm{P}<0.01)$ at $48 \mathrm{~h}$. Although the percentage of $\mathrm{G} 0 / \mathrm{G} 1$ phase cells increased in the combination-group compared with control-group $(\mathrm{P}<0.01)$, significant differences were not found in the combination-group when compared with MYC-KL-group or sCD40L-group ( $\mathrm{P}>0.05)$. The percentage of $\mathrm{G} 2 / \mathrm{M}$ phase cells in MYC-KL-group and sCD40L-group showed a declining trend compared with pCMV6-group and control-group respectively, but the differences did not reach statistical significance $(\mathrm{P}>0.05)$ at $48 \mathrm{~h}$. The percentage of $\mathrm{G} 2 / \mathrm{M}$ phase cells decreased in the combination-group compared with control-group $(\mathrm{P}<0.05)$ at $48 \mathrm{~h}$, but no significant differences were found when compared with MYC-KL-group or sCD40L-group. The percentage of $\mathrm{S}$ phase cells in each group showed no significant differences (Fig. 3A). The percentage of apoptosis increased in MYC-KL-group and SCD40L-group compared with pCMV6-group and control-group respectively $(\mathrm{P}<0.01)$ at $48 \mathrm{~h}$ after treatment, while the percentage of apoptosis in the combined-group showed further increased compared with MYC-KL-group or sCD40L-group respectively $(\mathrm{P}<0.05)$ (Fig. 3C). We did not find differences in apoptosis and cell cycle between MYC-KL group and SCD40L group. These data indicated that the combination can trigger A549 cells apoptosis more effectively.

Apoptosis-related gene Bax and Bcl-2 expression. We then investigated the potential pathways involved in the combined treatment-induced apoptosis. The results showed that Bcl-2, Bax and $\beta$-actin cDNA are 400 bp, 91 and 205 bp respectively, which is consistent with the expected results (Fig. 4B, and D). Although the anti-apoptosis gene $\mathrm{Bcl}-2$ was found down-regulated in MYC-KL-group and sCD40L-group 
compared with pCMV6-group and control-group, respectively $(\mathrm{P}<0.05)$ at $48 \mathrm{~h}$ after treatment, the Bcl-2 mRNA in the combination group was down-regulated compared with MYC-KL-group or sCD40L-group, respectively $(\mathrm{P}<0.05)$ (Fig. 4A). In contrast, Bax mRNA was up-regulated in MYC-KL-group and the combination-group compared with sCD40L-group or control-group or pCMV6-group $(\mathrm{P}<0.01)$; but a corresponding up-regulation was not found in $\mathrm{SCD} 40 \mathrm{~L}$ group ( $\mathrm{P}>0.05$, vs. control) (Fig. 4C). The results showed that Bcl-2 and Bax-related apoptosis pathways may be involved in the combined treatment-induced apoptosis.

\section{Discussion}

The traditional treatments for cancer include surgery, radiotherapy, and chemotherapy. Although these methods have good effects on patients in the short term, the defects including side effects, serious mutilation and high recurrence rate, can not be ignored. Moreover, in patients who have metastasis it is difficult to achieve a good long-term effect using traditional treatments (1). With the discovery of oncogenes and tumor suppressor genes, gene therapy has become increasingly prominent. Gene therapy include: immunogene therapy, tumor suppressor gene therapy, antisense gene therapy and suicide gene therapy. Mechanisms of gene therapy are divided into two categories: regulating the immune system; attacking tumor cells using the differences between tumor cells and normal cells. Since the tumor is a result of multi-factors, multi-links, and multi-stages, to correct it with a single factor to inhibit tumor is not effective. The ideal cancer therapy should have the potency to eradicate systemically at multiple sites as well as the specificity to discriminate between malignant and normal tissue. Considering these factors, combined gene therapy has become a research focus and has emerged as a potentially plausible approach for the control of cancers $(2,3)$.

In this study, we compared the effect of klotho gene, sCD40L and their combination on A549 cells. Although the effect of sCD40L on A549 cells has already been reported, there are few data on the effect of klotho gene, in addition, there are no data on the effect of their combination on A549 cells. Both klotho gene and SCD40L are thought to be tumor inhibition factors and their combination should have a cumulative regenerating effect on A549 cells. The major end-point of this study was to investigate if their combination has a synergistic or antagonistic effect on A549 cells.

sCD40L, a member of the tumor necrosis factor (TNF) superfamily, is predominantly expressed on activated CD4 ${ }^{+}$ T lymphocyte surface (14). CD40, a member of the TNF receptor superfamily, which is expressed on a plethora of cell types, including endothelial cells, dendritic cells and normal B lymphocytes, plays a critical role in B-cell activation by providing cell survival signals via interaction with the CD40 ligand $(14,15)$. In addition to its expression in normal lymphoid cells, CD40 is also expressed in a variety of malignant cells, including lymphomas, leukemias, ovaries, liver, lung and breast $(17,18)$. It has been noted that in various carcinoma cell lines treated with a soluble form or membranepresented CD40L, including non-small cell lung cancer $(21,22,23,28)$, breast cancer $(24)$, epithelial cells $(25)$, ovarian cancer (26), and bladder cancer (27), sCD40L-CD40 can inhibit tumor cell growth and increase apoptosis, and the mechanisms included a direct mechanism involving rapid up-regulation of TNFR-associated factor (TRAF)3 protein that promotes the caspase-9/caspase-3-associated intrinsic apoptotic machinery (27) and a indirect effects involving enhancing anti-tumor immunity (22). In this study, we found that soluble CD40 ligand can increase A549 cell proliferation inhibition and the apoptosis rate by inhibiting cell cycle and down-regulating Bcl-2 gene expression, and these findings are consistent with previous studies (21-27).

Klotho has been characterized as systemic anti-aging hormone $(4,7)$. People exhibiting a polymorphic variant of klotho gene are more prone to osteoporosis $(29,30)$, arterial hypertension (31) and emphysema (32). The signal pathways of klotho include retention of the calcium channel TRPV5 (33), inhibiting insulin/insulin-like growth factor-1 receptors (IGF-1) $(9,10)$ and acting as an inhibitor of the Wnt signaling (11). As the IGF-1/insulin pathways and the Wnt signaling have important functions in many cancer pathogenesis, klotho is already a hotspot to be studied as an anti-tumor gene. Our data indicated that klotho can increase cell proliferation inhibition and the apoptosis rate by inhibiting cell cycle, up-regulating Bax gene expression and down-regulating Bcl-2 gene expression in A549 cell line. Wolf et al showed that overexpression of klotho gene reduced proliferation of breast cancer cells and inhibited ligand-dependent activation of the IGF-1 and insulin pathways in breast cancer cells (10). These authors also observed that heterozygosity for the KLOTHO-V allele was associated with increased breast and ovarian cancers risk in Ashkenazi Jewish women carriers of BRCA1 mutation (34). Lee et al indicated that epigenetic silencing of klotho may occur during the late phase of cervical tumorigenesis, and consequent functional loss of klotho as the secreted Wnt antagonist may contribute to aberrant activation of the canonical Wnt pathway in carvical carcinoma (12). These data suggest that klotho can behave as a potential tumor suppressor in different kinds of human cancers. These findings agree with our results.

Taken together, the present study suggest that pretreatment with the combination of klotho and SCD40L can inhibit the growth of lung cancer cells A549 and promote their apoptosis more effective than transfected with klotho gene or treated with sCD40L alone. Several possible mechanisms may explain the results. In our study, we found that although the anti-apoptosis gene $\mathrm{Bcl}-2$ was found down-regulated by klotho or $\mathrm{sCD} 40 \mathrm{~L}$, the Bcl-2 mRNA in the combination group was further down-regulated. We analyzed that the combination showed a synergistic inhibition of apoptosis-related gene, so the combination resulted in a stronger apoptosis-promoting response compared with cells that were transfected with klotho or treated with sCD40L alone. It has also been found that in the presence of protein synthesis inhibitor cycloheximide (CHX) $(35,36)$ or chemotherapeutic agents that inhibit protein synthesis (26), the pro-apoptosis properties of CD40 ligation in carcinomas can be dramatically enhanced. This observation suggests that CD40 transduces potent survival signals which counterbalance its apoptosis-inducing effects. It was reported that CD40 can regulate protein synthesis through PI3K/AKT/ mTOR and ERK, the signaling pathways that counteract the 
pro-apoptotic properties of CD40 stimulation in carcinoma cells. Inhibition of these pathways may enhance the susceptibility of carcinoma cells to CD40-induced cell death (27). Coincidentally, Wolf et al found that overexpression of klotho gene was associated with reduced phosphorylation of not only IGF-1R, but also its downstream targets: IRS, AKT, extracellular signal-regulated kinases (ERK)-1 and ERK-2 (10). Based on the above data, we hypothesized that klotho may inhibit AKT and ERK pathways, and then enhances the susceptibility of carcinoma cells to CD40L-induced cells death. The other possible mechanisms included that the combination can block the cell cycle synergistically and/or exist the combined pathways that promote apoptosis. All these hypotheses require the support of further study.

Dysfunction of immune system of cancer patients and the lack of immune recognition molecules on the surface of tumor cells lead to tumor cells escape from immune surveillance. CD40 ligand was found to markedly enhance the antitumor immunogenicity of cancer cells presented by dendritic cells (DCs). CD40 ligand up-regulated expression of major histocompatibility complex class I/II and costimulatory molecules on the surface of DCs, and increased interleukin (IL)-12, IL-6, IL-15 production by DCs that can induce of cytotoxic T lymphocyte (CTL) responses $(37,38)$. B cells activated through CD40 up-regulate surface expression of major histocompatibility complex and costimulatory molecules, produce IL-12, and exhibit potent antigen-presenting properties (39). CD40 expresses on a variety of malignant cells, and activation CD40 on these tumor cells up-regulates costimulatory molecules and then reduces immune escape of the tumor cells $(17,18)$. Klotho gene down-regulation leads to abnormal immunity, while overexpression can increase the number of B cells in bone marrow and peripheral blood, inhibits the atrophy of thymus and maintain the thymus cells (40). Based on the previous data, we speculate that the combination with klotho gene and $\mathrm{SCD} 40 \mathrm{~L}$ can enhance the activation DCs and B cells, anti-tumor immunity, and reduce immunity escape of tumors in vivo. Additional studies in vivo are required in order to gain a better understanding of the role of klotho combined with $\mathrm{SCD} 40 \mathrm{~L}$ in human lung cancer. Better knowledge of the combination activation in human lung cancer will give rise to new therapeutic strategies against human lung cancer.

\section{Acknowledgements}

This study was partly supported by the grants from the National Natural Science Foundation of China (No. 30971320), Foundation of Jiangsu Key Researchers in Medical Science (RC2007051), and Foundation of Jiangsu Health Department in Scientific Research (P200904).

\section{References}

1. Berhoune M, Banu E, Scotte F, Oudard S and Bonun B Therapeutic strategy for treatment of metastatic non-small cell lung cancer. Ann Pharmacother 42: 1640-1652, 2008.

2. Kyung-sun H, Won-Kyung C, Jinsang Y, et al: Adenovirusmediated interleukin-12 gene transfer combined with cytosine deaminase followed by 5-fluorocytosine treatment exerts potent antitumor activity in Renca tumor-bearing mice. BMC Cancer 5: 51-57, 2005.
3. Di Cristofano A, De Acetis M and Koff A: PTEN and p27KIP1 cooperate in prostate cancer tumor suppression in the mouse. Nat Gene 27: 2222-2241, 2001.

4. Kuro-o M, Matsumura Y, Aizawa H, et al: Mutation of the mouse klotho gene leads to a syndrome resembling ageing. Nature 390: 45-51, 1997.

5. Min D, Panoskaltsis-Mortari A, Kuro-o M, Hollander GA, Blazar BR and Weinberg KI: Sustained thymopoiesis and improvement in functional immunity induced by exogenous KGF administration in murine models of aging. Blood 109: 2529-2537, 2007.

6. Kawaguchi H, Manabe N, Miyaura C, Chikuda H, Nakamura K and Kuro-o M: Independent impairment of osteoblast and osteoclast differentiation in klotho mouse exhibiting low-turnover osteopenia. J Clin Invest 1044: 229-237, 1999.

7. Matsumura Y, Aizawa H, Shiraki-Iida T, et al: Identification of the human klotho gene and its two transcripts encoding membrane secreted klotho protein. Biochem Biophy Res Commun 243: 626-630, 1998.

8. Kurosu H, Ogawa Y, Miyoshi M, Yamamoto M, Nandi A and Rosenblatt KP: Regulation of fibroblast growth factor-23 signaling by klotho. J Biol Chem 281: 6120-6123, 2006.

9. Kurosu H, Yamamoto M, Clark JD, Pastor JV, Nandi A, Gurnani P and McGuinness OP: Suppression of aging in mice by the hormone Klotho. Science 309: 1829-1393, 2005.

10. Wolf I, Levanon S, Bose S, et al: Klotho: a tumor suppressor and a modulator of the IGF-1 and FGF pathways in human breast cancer. Oncogene 292: 1-12, 2008.

11. Liu H, Fergusson MM, Castilho RM, Liu J, Cao L, Chen J and Malide D: Augmented Wnt signaling in a mammalian model of accelerated aging. Science 317: 803-806, 2007.

12. Lee J, Jeong DJ, Kim JS, et al: The anti-aging gene KLOTHO is a novel target for epigenetic silencing in human cervical carcinoma. Mol Cancer 9: 109, 2010.

13. Chen B, Wang XL, Zhao WH and Wu JQ: Klotho inhibits growth and promotes apoptosis in human lung cancer cell line A549. J Exp Clin Cancer Res 29: 99, 2010.

14. Berwelt-Baildon MS, Vonderheide RH, Maecker B, et al: Human primary and memory cytotoxic $\mathrm{T}$ lymphocyte responses are efficiently induced by means of CD40-activated B cell as antigen-presenting cells: potential for clinical application. Blood 99: 3319-3325, 2002

15. Gordon J and Pound JD: CD40 and its ligand: central players in B lymphocyte survival, grown and differentiation. Blood Rev 9: 53-56, 1995.

16. Grewal IS and Flavell RA: The CD40 ligand: at the center of the immune universe? Immunology 16: 59-70, 1997.

17. Tong AW and Stone MJ: Prospects for CD40-directed experimental therapy of human cancer. Cancer Gene Ther 10: 1-13, 2007.

18. Loskog AS and Eliopoulos AG: The Janus faces of CD40 in cancer. Semin Immunol 21: 301-307, 2009.

19. Johnson PW, Watt SM, Betts DR, Davies D, Jordan S, Norton AJ and Lister TA: Isolated follicular lymphoma cell are resistant to apoptosis and can be grown in vitro in the CD40/stromal cell system. Blood 82: 1848-1857, 1993.

20. Clod K, Asgari Z, Younes A, Palmer JL, Cabanillas F, Carbone A, Andreeff M and Younes A: Expression of CD40 ligand (CD154) in B and T lymphocytes of Hodgkin disease: potential therapeutic significance. Cancer 94: 1-5, 2002.

21. Yamada M, Shiroko T and Kawaguchi Y: CD40-CD40 lidand (CD154) engagement is required but not sufficient for modulating MHC class I, ICAM-I and Fas expression and proliferation of human non-small cell lung tumors. Int J Cancer 92: 589-599, 2001.

22. Noguchi M, Imaizumi K and Kawabe T: Indution of antitumor immunity by transduction of CD40 ligand gene and interferongamma gene into lung cancer. Cancer Gene Ther 8: 421-429, 2001.

23. Wu JQ, Zhao WH, Li Y, Zhu B and Yin KS: Adeno-associated virus mediated gene transfer into lung cancer cells promoting CD40 ligand-based immunotherapy. Virology 368: 309-316, 2007.

24. Tong AW, Papayoti MH, Netto G, Armstrong DT, Ordonez G, Lawson JM and Stone MJ: Growth-inhibitory effects of CD40 ligand (CD154) and its endogenous expression in human breast cancer. Clin Cancer Res 7: 691-703, 2001.

25. Eliopoulos AG, Dawson CW, Mosialos G, et al: CD40-induced growth inhibition in epithelial cell is mimicked by Epstein-Barr Virus-encoded LMP1: involvement of TRAF3 as a common mediator. Oncogene 13: 2243-2254, 1996. 
26. Schlienger K, Chu CS, Woo EY, et al: TRANCE-and CD40 ligand-matured dendritic cells reveal MHC I-restricted T cells specific for autologous tumor in late-stage ovarian cancer patients. Clin Cancer Res 9: 1517-1527, 2003.

27. Georgopoulos NT, Steele LP, Thomson MJ and Selby PJ: A novel mechanism of CD401-induced of carcinoma cells involving TRAF3 and JNK/AP-1 activation. Cell Death Differ 13: 1789-1801, 2006.

28. Chen WX, Chen YQ, Zou XS, et al: The mechanism of inhibiting lung cancer cell by CD40 ligand activations. Pract J Cancer 24: 132-134, 2009.

29. Mulin BH, Wilson SG, Fislam FM, Calautti M, Dick IM, Devine A and Prince RL: Klotho gene polymorphisms are associated with osteocalcin levels but not bone density of aged postmenopausal women. Calcif Tissue Int 77: 145-151, 2005.

30. Yamada Y, Ando F, Niino N and Shimokata H: Association of polymorphisms of the androgen receptor and klotho gene with bone mineral density in Japanese women. J Mol Med 83: 50-57, 2005.

31. Arking DE, Becker DM, Yanek LR, Fallin D, Judge DP, Moy TF, Bbecker LC and Dietz HC: KLOTHO allele status and the risk of early-onset occult coronary artery disease. Am J Hum Genet 72: 1154-1161, 2003

32. Suga T, Kurabayashi M, Sando Y, et al: Disruption of the klotho gene cause pulmonary emphysema in mice. Defect in maintenance of pulmonary integrity during postnatal life. Am J Respir Cell Mol Biol 22: 26-33, 2000.

33. Cha SK, Ortega B, Kurosu H, Rosenblatt KP, Kuro-o M and Huang CL: Removal of sialic acid involving klotho causes cellsurface retention of TRPV5 channel via binding to galectin-1. Proc Natl Acad Sci USA 105: 9805-9810, 2008.
34. Wolf I, Laitman Y, Rubinek T, et al: Functional variant of KLOTHO: a breast cancer risk modifier among BRCA1 mutation carriers of Ashkenazi origin. Oncogene 29: 26-23, 2010.

35. Eliopoulos AG, Davies C, Knox PG, Gallagher NJ, Afford SC, Adams DH and Young LS: CD40 induces apoptosis in carcinoma cells through an activation of cytotoxic ligand. Mol Cell Biol 20: 5503-5515, 2000.

36. Ghamande S, Hylander BL, Oflazoglu E, Lele S, Fanslow W and Repasky EA: Recombinant CD40 ligand therapy has significant antitumor effects on CD40-positive ovarian tumor xenografts grown in SCID mice and demonstrates an augmented effect with cisplatin. Cancer Res 61: 7556-7562, 2001.

37. Hoffmann TK, Meidenbauer N, Muller-Berghaus J, Storkus WJ and Whiteside TL: Proinflammatory cytokines and CD40 ligand enhance cross-presentation and cross-priming capability of human dendritic cells internalizing apoptotic cancer cells. J Immunother 24: 162-171, 2001.

38. Mazouz N, Ooms A, Moulin V, Van Meirvenne S, Uyttenhove C and Degiovanni G: CD40 triggering increases the efficiency of dendritic cells for antitumoral immunization. Cancer Immun 2: $2-10,2002$.

39. Li Q, Grover AG, Donald EJ, et al: Simultaneous targeting of CD3 on T cells and CD40 on B or dendritic cells augments the atitumor reactivity of tumor-primed lymph node cell. J Immunol 175: 1424-1432, 2005.

40. Arking DE, Krebsova A, Macek M, Macek M Sr, Macek M Jr, Aking A and Mian IS: Association of human aging with a functional variant of klotho. Proc Natl Acad Sci USA 99: 856-861, 2002. 